\title{
Blow-Up of Solutions for a Class of Sixth Order Nonlinear Strongly Damped Wave Equation
}

\author{
Huafei Di and Yadong Shang \\ School of Mathematics and Information Science, Guangzhou University, Guangzhou, Guangdong 510006, China \\ Correspondence should be addressed to Yadong Shang; gzydshang@126.com
}

Received 28 February 2014; Revised 1 May 2014; Accepted 4 May 2014; Published 15 May 2014

Academic Editor: Zhijun Liu

Copyright (C) 2014 H. Di and Y. Shang. This is an open access article distributed under the Creative Commons Attribution License, which permits unrestricted use, distribution, and reproduction in any medium, provided the original work is properly cited.

We consider the blow-up phenomenon of sixth order nonlinear strongly damped wave equation. By using the concavity method, we prove a finite time blow-up result under assumptions on the nonlinear term and the initial data.

\section{Introduction}

It is well known that nonlinear strongly damped wave equation is proposed to describe all kinds of viscous vibration system. The global well-posedness of third order nonlinear strongly damped wave equation

$$
u_{t t}-\alpha \Delta u_{t}-\Delta u=f(u), \quad \alpha>0, x \in \Omega, t>0,
$$

was studied by Webb [1] firstly. He gave the existence and asymptotic behavior of strong solutions for the problem (1). Then this result was improved by Y. C. Liu and D. C. Liu [2]. The existence and uniqueness of strong solutions were proved under the hypothesis of the weaker conditions. For two classes of strongly damped nonlinear wave equation, the finite time blow-up of solutions was proved by Shang [3]. A number of authors (Chen et al. [4], Zhou [5], and Al'shin et al. [6]) have shown the existence of the global weak solutions and the global attractors for third order nonlinear strongly damped wave equation.

For the fourth order nonlinear strongly damped wave equation, there are also some results about initial boundary value problem or Cauchy problem [7-9]. In [7], Shang studied the initial boundary value problem of the following equation:

$$
u_{t t}-\Delta u-\Delta u_{t}-\Delta u_{t t}=f(u), \quad x \in \Omega, t>0 .
$$

Under some assumptions on $f$ and $n=1,2,3$, he investigated the existence, uniqueness, asymptotic behavior, and blow-up phenomenon of the solutions.
In [8], $\mathrm{Xu}$ et al. considered the initial boundary value problem of fourth order wave equation with viscous damping term

$$
u_{t t}-\alpha u_{x x t}+u_{x x x x}=f\left(u_{x}\right)_{x}, \quad x \in \Omega, t>0 .
$$

They proved the global existence and nonexistence of the solution by argument related to the potential well-convexity method.

In order to investigate the water wave problem with surface tension, Schneider and Wayne [10] studied a class of Boussinesq equation as follows:

$$
u_{t t}=u_{x x}+u_{x x t t}+\mu u_{x x x x}-u_{x x x x t t}+\left(u^{2}\right)_{x x},
$$

where $x, t, \mu \in \mathbf{R}$. This type of equations can be formally derived from the $2 \mathrm{D}$ water wave problem and models the water wave problem with surface tension. They proved that the long wave limit can be described approximately by two decoupled Kawahara equations. A more natural model seems to be an extension from the classical Boussinesq equation as follows (see [11]):

$$
u_{t t}=u_{x x}+(\mu+1) u_{x x x x}-u_{x x x x x x}+\left(u^{2}\right)_{x x} .
$$

Wang and $\mathrm{Mu}$ [12] studied the Cauchy problem of the equation

$$
u_{t t}=u_{x x}+u_{x x t t}-u_{x x x x}-u_{x x x x t t}+f(u)_{x x} .
$$


They obtained the existence and uniqueness of the local solutions and proved the blow-up of solutions to the problem (6). Esfahani et al. [13] studied the solutions of

$$
u_{t t}=u_{x x}+\beta u_{x x x x}+u_{x x x x x x}+\left(|u|^{\alpha} u\right)_{x x}
$$

where $\beta= \pm 1$ and $\alpha>0$. They proved the local wellposedness in $L^{2}(R)$ and $H^{1}(R)$ and gave finite time blow-up results to the problem (7).

For the sixth order nonlinear wave equation with strong damping term

$$
u_{t t}-\Delta u-v \Delta u_{t}+\Delta^{2} u+\Delta^{2} u_{t t}=\Delta f(u),
$$

H. W. Wang and S. B. Wang [14] established a global existence result of small amplitude solutions of the Cauchy problem (8) for all space dimensions $n>1$. When $v=0, H$. W. Wang and S. B. Wang [15] studied the long-time behavior of small solutions of the Cauchy problem for a Rosenau equation. The decay and scattering for small amplitude solution are established.

In this paper, we study a class of sixth order nonlinear strongly damped wave equation:

$$
\begin{gathered}
u_{t t}-\Delta u_{t}=\operatorname{div} \vec{\sigma}(\nabla u)+\Delta u+\mu \Delta^{2} u+\Delta^{3} u, \quad x \in \Omega, t>0, \\
u(x, 0)=u_{0}(x), \quad u_{t}(x, 0)=u_{1}(x), \quad x \in \Omega, \\
\left(\mathscr{B}_{i} u\right)(x, t)=0, \quad x \in \partial \Omega, t>0,
\end{gathered}
$$

where $\mu>0, \Omega$ is a bounded domain of $R^{n}(n \geq 1)$ with a smooth boundary $\partial \Omega$, and $\mathscr{B}_{i}, i=0,1,2$, are homogeneous boundary condition:

$$
D=\left\{u \in C^{6}(\bar{\Omega}) \mid \mathscr{B}_{i} u=\nabla^{i} u \equiv 0, i=0,1,2, x \in \partial \Omega\right\} .
$$

By using the ideas of the concavity theory introduced by Levine [17], we prove the finite time blow-up results under assumption on the nonlinear term $\operatorname{div} \vec{\sigma}(\nabla u)$ and the initial data $u_{0}, u_{1}$.

\section{Preliminaries and Main Results}

In this section, we introduce some notations, basic ideas, and important lemmas which will be needed in the course of the paper.

Let $H=\mathscr{L}^{2}(\Omega)$ be a Hilbert space which is equipped with the scalar product $(u, v)=\int_{\Omega} u(x) v(x) d x$.

Now, we define

$$
A u=-\Delta u-\mu \Delta^{2} u-\Delta^{3} u
$$

where $A: D \rightarrow H$ is a symmetric linear operator and satisfies $(u, A v)=(A u, v)$ for all $u, v \in D \subseteq H$.

For the nonlinear term $\operatorname{div} \vec{\sigma}(\nabla u)$ of the problem (9), $\vec{\sigma}=\left(\sigma_{1}, \sigma_{2}, \ldots, \sigma_{n}\right): D^{n} \rightarrow H^{n}$ is a vector function which satisfies the following conditions. (a) Assume that the Fréchet derivative $\vec{\sigma}_{x}$ is a symmetric, bounded, linear operator on $H^{n}$ and that $x \rightarrow \vec{\sigma}_{x}$ is a continuous map from $D^{n}$ to $\mathfrak{Q}(H)^{n}$.

(b) The scalar valued function $G: D \rightarrow \mathrm{R}$ is defined by

$$
\begin{aligned}
G(u) & =\int_{0}^{1}(\operatorname{div} \vec{\sigma}(\rho \nabla u), u) d \rho \\
& =\int_{\Omega}\left(\int_{0}^{1} \operatorname{div} \vec{\sigma}(\rho \nabla u) u d \rho\right) d x \\
& =\int_{\Omega}\left(\int_{0}^{1} \frac{d}{d \rho}\left(\int_{0}^{\rho u} \operatorname{div} \vec{\sigma}(\nabla z) d z\right) d \rho\right) d x \\
& =\int_{\Omega}\left(\int_{0}^{u} \operatorname{div} \vec{\sigma}(\nabla z) d z\right) d x,
\end{aligned}
$$

where $G(u)$ denotes the potential associated with $\operatorname{div} \vec{\sigma}(\nabla u)$. The Fréchet derivative of $G(u)$ is $G_{u}$ which can be shown to act as follows:

$$
G_{u} v=(\operatorname{div} \vec{\sigma}(\nabla u), v),
$$

for all $u, v \in D$.

(c) Assume that for some $\alpha>0$

$$
(\operatorname{div} \vec{\sigma}(\nabla u), u) \geq 2(2 \alpha+1) G(u),
$$

for all $u \in D$.

To obtain the finite time blow-up result, we need the following interpolation inequality of Evance [16] for function in $W_{0}^{k, p}(\Omega)$.

Lemma 1. For all $u \in W_{0}^{k, p}(\Omega)$, if $1 \leq p<\infty, j$ and $k$ are integers, and $1 \leq j \leq k,(j / k) \leq \alpha \leq 1$, then

$$
\left\|D^{j} u\right\|_{L^{q}(\Omega)} \leq C\left\|D^{k} u\right\|_{L^{p}(\Omega)}^{\alpha}\|D u\|_{L^{r}(\Omega)}^{1-\alpha}, \quad \forall u \in W_{0}^{k, p}(\Omega),
$$

where $(1 / q)-(j / n)=\alpha((1 / p)-(k / n))+(1-\alpha)((1 / r)-(1 / n))$, with the constant $C$ depending only on $(p, r, j, k, \alpha, n)$ and $\Omega$.

In particular, if $j=2, k=3$, and $p=q=r=2$, one has

$$
\left\|D^{2} u\right\|_{L^{2}(\Omega)} \leq C\left\|D^{3} u\right\|_{L^{2}(\Omega)}^{\alpha}\|D u\|_{L^{2}(\Omega)}^{1-\alpha}, \quad \forall u \in W_{0}^{3,2}(\Omega) .
$$

Lemma 2. Assume that $u \in W_{0}^{3,2}(\Omega)$ and $0<\mu \leq$ $\left(1 / C_{1}\right)$ (with $C_{1}$ depending on the constant $C$ of Sobolev's interpolation inequality); then $(A u, u) \geq 0$.

Proof. By Lemma 1, we see that

$$
\left\|D^{2} u\right\|_{L^{2}(\Omega)} \leq C\left\|D^{3} u\right\|_{L^{2}(\Omega)}^{\alpha}\|D u\|_{L^{2}(\Omega)}^{1-\alpha}, \quad \forall u \in W_{0}^{3,2}(\Omega) .
$$

Using Young's inequality, we have

$$
\begin{aligned}
\left\|D^{2} u\right\|_{L^{2}(\Omega)}^{2} & \leq C\left\|D^{3} u\right\|_{L^{2}(\Omega)}^{2 \alpha}\|D u\|_{L^{2}(\Omega)}^{2(1-\alpha)} \\
& \leq C\left[\alpha\left\|D^{3} u\right\|_{L^{2}(\Omega)}^{2}+(1-\alpha)\|D u\|_{L^{2}(\Omega)}^{2}\right] \\
& \leq C_{1}\left[\left\|D^{3} u\right\|_{L^{2}(\Omega)}^{2}+\|D u\|_{L^{2}(\Omega)}^{2}\right] .
\end{aligned}
$$


For the operator $A$, using integration of parts, we have

$$
\begin{aligned}
(A u, u) & =\left(-\Delta u-\mu \Delta^{2} u-\Delta^{3} u, u\right) \\
& =\|\nabla u\|_{L^{2}(\Omega)}^{2}+\left\|\nabla^{3} u\right\|_{L^{2}(\Omega)}^{2}-\mu\left\|\nabla^{2} u\right\|_{L^{2}(\Omega)}^{2} \\
& \geq \frac{1}{C_{1}}\left\|\nabla^{2} u\right\|_{L^{2}(\Omega)}^{2}-\mu\left\|\nabla^{2} u\right\|_{L^{2}(\Omega)}^{2} \\
& =\left(\frac{1}{C_{1}}-\mu\right)\left\|\nabla^{2} u\right\|_{L^{2}(\Omega)}^{2},
\end{aligned}
$$

where $0<\mu \leq\left(1 / C_{1}\right)$.

The verification of the action of $G_{x}$ can be proved from the definition. The details, not being germane to this paper, are omitted here. But a formula will be useful in the sequel as follows.

Lemma 3. Let $G(u)=\int_{0}^{1}(\operatorname{div} \sigma(\nabla(\rho u)), u) d \rho$; then one has

$$
G(u(t))-G(u(0))=\int_{0}^{t}\left(\operatorname{div} \sigma(\nabla u(\eta)), u_{\eta}(\eta)\right) d \eta,
$$

for $u:[0, T) \rightarrow D$ with a strongly continuous derivative $u_{t}$. Proof. By the chain rule and the action of $G_{x}$, we have

$$
\begin{aligned}
\frac{d}{d t} G(u) & =\frac{d}{d t} \int_{0}^{1}(\operatorname{div} \vec{\sigma}(\nabla(\rho u)), u) d \rho \\
& =-\frac{d}{d t} \int_{0}^{1}(\vec{\sigma}(\nabla(\rho u)), \nabla u) d \rho \\
& =-\int_{0}^{1} \rho\left(\vec{\sigma}_{\rho \nabla u} \cdot \nabla u_{t}, \nabla u\right)+\left(\vec{\sigma}(\rho \nabla u), \nabla u_{t}\right) d \rho \\
& =-\int_{0}^{1} \rho\left(\vec{\sigma}_{\rho \nabla u} \cdot \nabla u, \nabla u_{t}\right)+\left(\vec{\sigma}(\rho \nabla u), \nabla u_{t}\right) d \rho \\
& =-\int_{0}^{1} \rho \frac{d}{d \rho}\left(\vec{\sigma}(\rho \nabla u), \nabla u_{t}\right)+\left(\vec{\sigma}(\rho \nabla u), \nabla u_{t}\right) d \rho \\
& =-\int_{0}^{1} \frac{d}{d \rho}\left[\rho\left(\vec{\sigma}(\rho \nabla u), \nabla u_{t}\right)\right] d \rho \\
& =\left(\operatorname{div} \vec{\sigma}(\nabla u), u_{t}\right),
\end{aligned}
$$

where we have used the symmetry of $\vec{\sigma}_{x}$ in the fourth line.

The following lemma contributing to the result of this paper is analogous to Corollary 1.1 of [17] with slight modification.
Lemma 4. Assume that $\vec{\sigma}$ is homogenous of degree $1+\gamma$ for some $\gamma>0$ (i.e., $\vec{\sigma}(s h)=s^{1+\gamma} \vec{\sigma}(h)$ for all $s>0$ and for all $h \in D)$. Let $\left(h_{0}, \operatorname{div} \vec{\sigma}\left(\nabla h_{0}\right)\right)>0$ for some $h_{0} \in D$. Then there are infinitely many vectors $u_{0}$ such that

$$
\begin{aligned}
G\left(u_{0}\right) & >\frac{1}{2}\left(u_{0}, A u_{0}\right) \\
& =\frac{1}{2}\left[\left\|\nabla^{3} u_{0}\right\|_{L^{2}(\Omega)}^{2}+\left\|\nabla u_{0}\right\|_{L^{2}(\Omega)}^{2}-\mu\left\|\nabla^{2} u_{0}\right\|_{L^{2}(\Omega)}^{2}\right] .
\end{aligned}
$$

Proof. Let $u_{0}=s h_{0}$, where $s$ is large enough so that

$$
\begin{aligned}
s^{\gamma} G\left(h_{0}\right) & =s^{\gamma} \int_{0}^{1}\left(\operatorname{div} \vec{\sigma}\left(\rho \nabla h_{0}\right), h_{0}\right) d \rho \\
& >\frac{1}{2}\left(h_{0}, A h_{0}\right) \\
& =\frac{1}{2}\left[\left\|\nabla^{3} h_{0}\right\|_{L^{2}(\Omega)}^{2}+\left\|\nabla h_{0}\right\|_{L^{2}(\Omega)}^{2}-\mu\left\|\nabla^{2} h_{0}\right\|_{L^{2}(\Omega)}^{2}\right] .
\end{aligned}
$$

Then for all

$$
s>s_{0}=\sqrt[\gamma]{\frac{(2+\gamma)\left[\left\|\nabla^{3} h_{0}\right\|_{L^{2}(\Omega)}^{2}+\left\|\nabla h_{0}\right\|_{L^{2}(\Omega)}^{2}-\mu\left\|\nabla^{2} h_{0}\right\|_{L^{2}(\Omega)}^{2}\right]}{2\left(\operatorname{div} \vec{\sigma}\left(\nabla h_{0}\right), h_{0}\right)}},
$$

we have

$$
\begin{aligned}
G\left(u_{0}\right) & =\int_{0}^{1}\left(\operatorname{div} \vec{\sigma}\left(\rho s \nabla h_{0}\right), s h_{0}\right) d \rho \\
& =-\int_{0}^{1}\left(\vec{\sigma}\left(\rho s \nabla h_{0}\right), s \nabla h_{0}\right) d \rho \\
& =s^{2+\gamma} G\left(h_{0}\right)>\frac{1}{2}\left(u_{0}, A h_{0}\right) .
\end{aligned}
$$

The local existence of solution for the problem (9) can be obtained by the standard Faedo-Galerkin approximation methods. The interested reader is referred to Lions [18] or Robinson [19] for details.

Next, we are ready to state the blow-up result of this paper.

Theorem 5. Let $u:[0, T) \rightarrow D$ be a strongly continuously differentiable solution of (9) in the D norm. Suppose that $0<$ $\mu \leq\left(1 / C_{1}\right)$ with $\left(C_{1}\right.$ depending on the constant $C$ of Sobolev's interpolation inequality) (div $\vec{\sigma}(\nabla u), u) \geq 2(2 \alpha+1) G(u)$, where $\left(G(u)=\int_{0}^{1}(\operatorname{div} \vec{\sigma}(\rho \nabla u), u) d \rho\right)$. Finally let $u_{0}$ satisfy

$$
\begin{aligned}
G\left(u_{0}\right) & >\frac{1}{2}\left(u_{0}, A u_{0}\right) \\
& =\frac{1}{2}\left[\left\|\nabla^{3} u_{0}\right\|_{L^{2}(\Omega)}^{2}+\left\|\nabla u_{0}\right\|_{L^{2}(\Omega)}^{2}-\mu\left\|\nabla^{2} u_{0}\right\|_{L^{2}(\Omega)}^{2}\right] .
\end{aligned}
$$


Then the solution u can only exist on a bounded interval $[0, T)$, and in fact

$$
\begin{aligned}
T \leq & \left(4 \alpha^{2} \beta\left(u_{0}, u_{0}\right)\right. \\
& +\left[\left(\nabla u_{0}, \nabla u_{0}\right)-2 \alpha\left(u_{0}, u_{1}\right)\right. \\
& \left.\left.+\sqrt{\left[\left(\nabla u_{0}, \nabla u_{0}\right)-2 \alpha\left(u_{0}, u_{1}\right)\right]^{2}+4 \alpha^{2} \beta\left(u_{0}, u_{0}\right)}\right]^{2}\right) \\
& \times\left(4 \alpha ^ { 2 } \beta \left(\left[\left(\nabla u_{0}, \nabla u_{0}\right)-2 \alpha\left(u_{0}, u_{1}\right)\right]^{2}\right.\right. \\
& \left.\left.+4 \alpha^{2} \beta\left(u_{0}, u_{0}\right)\right)^{1 / 2}\right)^{-1},
\end{aligned}
$$

while also

$$
\lim _{t \rightarrow T^{-}}\left[\|u\|_{L^{2}(\Omega)}^{2}+\int_{0}^{t}\|\nabla u\|_{L^{2}(\Omega)}^{2} d \eta\right]=+\infty
$$

and consequently

$$
\limsup _{t \rightarrow T^{-}}\|u\|_{H^{1}}^{2}=+\infty
$$

Proof. For arbitrary $T_{0}, \beta, \tau>0$ and $t \in\left[0, T_{0}\right)$, let

$$
\begin{aligned}
F(t)= & (u, u)+\int_{0}^{t}(u,-\Delta u) d \eta \\
& +\left(T_{0}-t\right)\left(u_{0},-\Delta u_{0}\right)+\beta(t+\tau)^{2} .
\end{aligned}
$$

A direct computation yields

$$
\begin{aligned}
F^{\prime}(t) & =2\left(u, u_{t}\right)+(u,-\Delta u)-\left(u_{0},-\Delta u_{0}\right)+2 \beta(t+\tau) \\
& =2\left(u, u_{t}\right)+(\nabla u, \nabla u)-\left(\nabla u_{0}, \nabla u_{0}\right)+2 \beta(t+\tau) \\
& =2\left(u, u_{t}\right)+2 \int_{0}^{t}\left(\nabla u, \nabla u_{\eta}\right) d \eta+2 \beta(t+\tau)
\end{aligned}
$$

Suppressing the argument $t$, we see that

$$
\begin{aligned}
F^{\prime \prime}(t)= & 2\left(u, u_{t t}\right)+2\left(u_{t}, u_{t}\right) \\
& +2 \int_{0}^{t}\left(\nabla u, \nabla u_{\eta}\right)_{\eta} d \eta+2\left(\nabla u_{1}, \nabla u_{0}\right)+2 \beta
\end{aligned}
$$

Hence, from (30), (31), and (32), we find after some algebra that

$$
\begin{aligned}
F^{\prime \prime} F- & (\alpha+1)\left(F^{\prime}(t)\right)^{2} \\
= & 4(\alpha+1) S^{2}+2 F\left[\left(u, u_{t t}\right)-(2 \alpha+1)\left(u_{t}, u_{t}\right)\right] \\
& +2 F\left[\int_{0}^{t}\left(\nabla u, \nabla u_{\eta}\right)_{\eta} d \eta-2(\alpha+1) \int_{0}^{t}\left(\nabla u_{\eta}, \nabla u_{\eta}\right) d \eta\right] \\
& +4(\alpha+1)\left(T_{0}-t\right)\left(\nabla u_{0}, \nabla u_{0}\right) \\
& \times\left[\left(u_{t}, u_{t}\right)+\int_{0}^{t}\left(\nabla u_{\eta}, \nabla u_{\eta}\right) d \eta+\beta\right] \\
& +2 F\left[\left(\nabla u_{1}, \nabla u_{0}\right)-(2 \alpha+1) \beta\right],
\end{aligned}
$$

where

$$
\begin{aligned}
S^{2}= & -\left[\left(u, u_{t}\right)+\int_{0}^{t}\left(\nabla u, \nabla u_{\eta}\right) d \eta+\beta(t+\tau)\right]^{2} \\
& +\left[(u, u)+\int_{0}^{t}(u,-\Delta u) d \eta+\beta(t+\tau)^{2}\right] \\
& \times\left[\left(u_{t}, u_{t}\right)+\int_{0}^{t}\left(\nabla u_{\eta}, \nabla u_{\eta}\right) d \eta+\beta\right] .
\end{aligned}
$$

Using Schwarz's inequality, we have

$$
\begin{gathered}
(u, u)\left(u_{t}, u_{t}\right) \geq\left(u, u_{t}\right)^{2} \\
\int_{0}^{t}(\nabla u, \nabla u) d \eta \int_{0}^{t}\left(\nabla u_{\eta}, \nabla u_{\eta}\right) d \eta \geq\left[\int_{0}^{t}\left(\nabla u, \nabla u_{\eta}\right) d \eta\right]^{2} \\
(u, u) \int_{0}^{t}\left(\nabla u_{\eta}, \nabla u_{\eta}\right) d \eta+\left(u, u_{t}\right) \int_{0}^{t}(\nabla u, \nabla u) d \eta \\
\geq 2 \sqrt{(u, u)\left(u_{t}, u_{t}\right) \int_{0}^{t}(\nabla u, \nabla u) d \eta \int_{0}^{t}\left(\nabla u_{\eta}, \nabla u_{\eta}\right) d \eta} \\
\geq 2\left(u, u_{t}\right) \int_{0}^{t}\left(\nabla u, \nabla u_{\eta}\right) d \eta, \\
(u, u) \beta+\left(u_{t}, u_{t}\right) \beta(t+\tau)^{2} \\
\geq 2 \sqrt{(u, u)\left(u_{t}, u_{t}\right) \beta^{2}(t+\tau)^{2}} \\
\geq 2\left(u, u_{t}\right) \beta(t+\tau) .
\end{gathered}
$$


By (35), we have $S^{2} \geq 0$. Let

$H(t)$

$$
\begin{aligned}
= & \left(u, u_{t t}\right)-(2 \alpha+1)\left(u_{t}, u_{t}\right)+\left(\nabla u_{1}, \nabla u_{0}\right)-(2 \alpha+1) \beta \\
& -\int_{0}^{t}\left(\Delta u_{\eta}, u\right)_{\eta} d \eta+2(\alpha+1) \int_{0}^{t}\left(\Delta u_{\eta}, u_{\eta}\right) d \eta \\
= & \left(u, u_{t t}\right)-(2 \alpha+1)\left(u_{t}, u_{t}\right)+\left(\nabla u_{1}, \nabla u_{0}\right)-(2 \alpha+1) \beta \\
& -\int_{0}^{t}\left(u_{\eta \eta}, u\right)_{\eta} d \eta+2(\alpha+1) \int_{0}^{t}\left(u_{\eta \eta}, u_{\eta}\right) d \eta \\
& -\int_{0}^{t}\left[(A u, u)_{\eta}-2(\alpha+1)\left(A u, u_{\eta}\right)\right] d \eta \\
& +\int_{0}^{t}\left[(\operatorname{div} \vec{\sigma}(\nabla u), u)_{\eta}-2(\alpha+1)\left(\operatorname{div} \vec{\sigma}(\nabla u), u_{\eta}\right)\right] d \eta .
\end{aligned}
$$

Thus

$$
\begin{aligned}
H^{\prime}(t) & \\
= & -2 \alpha\left(u_{t t}, u_{t}\right)+2 \alpha\left(u_{t}, A u\right) \\
& +(\operatorname{div} \vec{\sigma}(\nabla u), u)_{t}-2(\alpha+1) \frac{d}{d t} G(u) \\
= & -2 \alpha\left(\Delta u_{t}, u_{t}\right)-2 \alpha\left(\operatorname{div} \vec{\sigma}(\nabla u), u_{t}\right) \\
& +4 \alpha\left(u_{t}, A u\right)+(\operatorname{div} \vec{\sigma}(\nabla u), u)_{t}-2(\alpha+1) \frac{d}{d t} G(u) \\
= & 2 \alpha\left(\nabla u_{t}, \nabla u_{t}\right)+4 \alpha\left(u_{t}, A u\right) \\
& +(\operatorname{div} \vec{\sigma}(\nabla u), u)_{t}-(4 \alpha+2) \frac{d}{d t} G(u) .
\end{aligned}
$$

Using the positive semidefiniteness of $A$, Lemma 3, and (14), we have

$$
\begin{aligned}
H^{\prime}(t)= & H(0)+2 \alpha \int_{0}^{t}\left(\nabla u_{\eta}, \nabla u_{\eta}\right) d \eta+4 \alpha \int_{0}^{t}\left(u_{\eta}, A u\right) d \eta \\
& +\int_{0}^{t}\left[(\operatorname{div} \vec{\sigma}(\nabla u), u)_{\eta}-(4 \alpha+2) \frac{d}{d \eta} G(u)\right] d \eta \\
= & (4 \alpha+2) G\left(u_{0}\right)-(2 \alpha+1)\left(u_{0}, A u_{0}\right) \\
& +\left(u_{1}, u_{1}\right)+\left(\nabla u_{1}, \nabla u_{1}\right)-(2 \alpha+1) \beta \\
& +2 \alpha \int_{0}^{t}\left(\nabla u_{\eta}, \nabla u_{\eta}\right) d \eta+2 \alpha(u, A u) \\
& +[(\operatorname{div} \vec{\sigma}(\nabla u), u)-(4 \alpha+2) G(u)]
\end{aligned}
$$

$$
\begin{aligned}
& \geq(4 \alpha+2) G\left(u_{0}\right)-(2 \alpha+1)\left(u_{0}, A u_{0}\right)-(2 \alpha+1) \beta \\
& =2(2 \alpha+1)\left[G\left(u_{0}\right)-\frac{1}{2}\left(A u_{0}, u_{0}\right)-\frac{\beta}{2}\right] .
\end{aligned}
$$

Thus, from what has been discussed above, we have

$$
\begin{aligned}
& F F^{\prime \prime}-(\alpha+1)\left(F^{\prime}\right)^{2} \\
& \quad \geq 4(2 \alpha+1) F\left[G\left(u_{0}\right)-\frac{1}{2}\left(A u_{0}, u_{0}\right)-\frac{\beta}{2}\right] .
\end{aligned}
$$

Therefore, for any $\beta>0$ such that

$$
\begin{aligned}
\beta \leq & 2 G\left(u_{0}\right)-\left(A u_{0}, u_{0}\right) \\
= & 2 \int_{0}^{1}\left(\operatorname{div} \vec{\sigma}\left(\rho \nabla u_{0}\right), u_{0}\right) d \rho \\
& -\left[\left\|\nabla^{3} u_{0}\right\|_{L^{2}(\Omega)}^{2}+\left\|\nabla u_{0}\right\|_{L^{2}(\Omega)}^{2}-\mu\left\|\nabla^{2} u_{0}\right\|_{L^{2}(\Omega)}^{2}\right],
\end{aligned}
$$

$F F^{\prime \prime}-(\alpha+1)\left(F^{\prime}\right)^{2} \geq 0$ and $\left(F^{-\alpha}(t)\right)^{\prime \prime} \leq 0$. We see that $F(t)>$ 0 , for all $t \in\left[0, T_{0}\right)$ and $F^{\prime}(0)=2\left(u_{0}, u_{1}\right)+2 \beta \tau>0$, if $\tau$ is sufficiently large. Since a concave function must always lie below any tangent line, so we have

$$
F^{-\alpha}(t) \leq F^{-\alpha}(0)+\left[F^{-\alpha}(0)\right]^{\prime} t
$$

or

$$
F(t) \geq F^{1+(1 / \alpha)}(0)\left[F(0)-\alpha t F^{\prime}(0)\right]^{-1 / \alpha} ;
$$

we may choose $T_{0}$ such that $T_{0} \geq\left(F(0) / \alpha\left(F^{\prime}(0)\right)\right) \equiv T_{\beta \tau}$. Thus, we see that the interval of existence of $u$ must be contained in $\left[0, F(0) / \alpha\left(F^{\prime}(0)\right)\right)$ and that the finite time blowup of solution of ( 9$)$ is proved. Let

$$
\begin{aligned}
\beta_{0}= & 2 G\left(u_{0}\right)-\left(A u_{0}, u_{0}\right) \\
= & 2 \int_{0}^{1}\left(\operatorname{div} \vec{\sigma}\left(\rho \nabla u_{0}\right), u_{0}\right) d \rho \\
& -\left[\left\|\nabla^{3} u_{0}\right\|_{L^{2}(\Omega)}^{2}+\left\|\nabla u_{0}\right\|_{L^{2}(\Omega)}^{2}-\mu\left\|\nabla^{2} u_{0}\right\|_{L^{2}(\Omega)}^{2}\right], \\
T_{\beta \tau}= & \frac{F(0)}{\alpha\left(F^{\prime}(0)\right)}=\frac{\left(u_{0}, u_{0}\right)+T_{0}\left(\nabla u_{0}, \nabla u_{0}\right)+\beta \tau^{2}}{2 \alpha\left(u_{0}, u_{1}\right)+2 \alpha \beta \tau} .
\end{aligned}
$$

Since $T_{0} \geq T_{\beta \tau}$, we have $\left(F^{-\alpha}(t)\right)^{\prime \prime} \leq 0$. Even if we take $T_{0}=$ $T_{\beta \tau}$, we have

$$
T_{\beta \tau}=\left[\left(u_{0}, u_{0}\right)+\beta \tau^{2}\right]\left[2 \alpha\left(u_{0}, u_{1}\right)+2 \alpha \beta \tau-\left(\nabla u_{0}, \nabla u_{0}\right)\right]^{-1}
$$


thus we must choose $\tau$ so large such that $2 \alpha \beta \tau>\left(\nabla u_{0}, \nabla u_{0}\right)-$ $2 \alpha\left(u_{0}, u_{1}\right)$. As a function, $T_{\beta \tau}$ has a minimum at

$$
\begin{aligned}
\tau= & \tau(\beta) \\
= & \left(\left(\nabla u_{0}, \nabla u_{0}\right)-2 \alpha\left(u_{0}, u_{1}\right)\right. \\
& \left.+\sqrt{\left[\left(\nabla u_{0}, \nabla u_{0}\right)-2 \alpha\left(u_{0}, u_{1}\right)\right]^{2}+4 \alpha^{2} \beta\left(u_{0}, u_{0}\right)}\right) \\
& \quad \times(2 \alpha \beta)^{-1},
\end{aligned}
$$

and this minimum is

$$
\begin{aligned}
& T_{\beta \tau(\beta)} \\
& =\left(4 \alpha^{2} \beta\left(u_{0}, u_{0}\right)\right. \\
& +\quad\left[\left(\nabla u_{0}, \nabla u_{0}\right)-2 \alpha\left(u_{0}, u_{1}\right)\right. \\
& \left.\left.\quad+\sqrt{\left[\left(\nabla u_{0}, \nabla u_{0}\right)-2 \alpha\left(u_{0}, u_{1}\right)\right]^{2}+4 \alpha^{2} \beta\left(u_{0}, u_{0}\right)}\right]^{2}\right) \\
& \quad \times\left(4 \alpha ^ { 2 } \beta \left(\left[\left(\nabla u_{0}, \nabla u_{0}\right)-2 \alpha\left(u_{0}, u_{1}\right)\right]^{2}\right.\right. \\
& \left.\left.+4 \alpha^{2} \beta\left(u_{0}, u_{0}\right)\right)^{1 / 2}\right)^{-1} .
\end{aligned}
$$

Since $\beta$ is restricted to $\left[0, \beta_{0}\right]$, we see that $T_{\beta \tau(\beta)}$ attains its minimum at $\beta=\beta_{0}$. Thus $T$ cannot exceed $T_{\beta \tau\left(\beta_{0}\right)}$.

\section{Conflict of Interests}

The authors declare that there is no conflict of interests regarding the publication of this paper.

\section{References}

[1] G. F. Webb, "Existence and asymptotic behavior for a strongly damped nonlinear wave equation," Canadian Journal of Mathematics, vol. 32, no. 3, pp. 631-643, 1980.

[2] Y. C. Liu and D. C. Liu, "The initial-boundary value problem for the equation $u_{t t}-\alpha \Delta u_{t}-\Delta u=f(u)$," Journal of Huazhong University of Science and Technology, vol. 16, no. 6, pp. 169-173, 1988.

[3] Y. D. Shang, "Blow-up of solutions for two classes of strongly damped nonlinear wave equations," Journal of Engineering Mathematics, vol. 17, no. 2, pp. 65-70, 2000.

[4] F. X. Chen, B. L. Guo, and P. Wang, "Long time behavior of strongly damped nonlinear wave equations," Journal of Differential Equations, vol. 147, no. 2, pp. 231-241, 1998.

[5] S. F. Zhou, "Attractors for strongly damped wave equation in uniform spaces," Nonlinear Analysis: Theory, Methods \& Applications, vol. 64, pp. 174-187, 2006.

[6] A. B. Al'shin, M. O. Korpusov, and A. G. Sveshnikov, Blow-Up in Nonlinear Sobolev Type Equations, vol. 15 of De Gruyter Series in Nonlinear Analysis and Applications, Walter de Gruyter, Berlin, Germany, 2011.
[7] Y. D. Shang, "Initial-boundary value problem for the equation $u_{t t}-\Delta u-\Delta u_{t}-\Delta u_{t t}=f(u), "$ Acta Mathematicae Applicatae Sinica, vol. 23, no. 3, pp. 385-393, 2000.

[8] R. Z. Xu, S. Wang, Y. B. Yang, and Y. H. Ding, "Initial boundary value problem for a class of fourth-order wave equation with viscous damping term," Applicable Analysis, vol. 92, no. 7, pp. 1403-1416, 2013.

[9] Y. C. Liu and R. Z. Xu, "Fourth order wave equations with nonlinear strain and source terms," Journal of Mathematical Analysis and Applications, vol. 331, no. 1, pp. 585-607, 2007.

[10] G. Schneider and C. E. Wayne, "Kawahara dynamics in dispersive media," Physica D: Nonlinear Phenomena, vol. 152-153, pp. 384-394, 2001.

[11] M. J. Boussinesq, Essai sur la Théorie des Eaux Courantes, vol. 3 of Mémoires Présentés par Divers Savans à l'Académie des Sciences de l'Institut de France (séries 2), 1877.

[12] Y. Wang and C. L. Mu, "Blow-up and scattering of solution for a generalized Boussinesq equation," Applied Mathematics and Computation, vol. 188, no. 2, pp. 1131-1141, 2007.

[13] A. Esfahani, L. G. Farah, and H. Wang, "Global existence and blow-up for the generalized sixth-order Boussinesq equation," Nonlinear Analysis: Theory, Methods \& Applications, vol. 75, no. 11, pp. 4325-4338, 2012.

[14] H. W. Wang and S. B. Wang, "Global existence and asymptotic behavior of solution for the Rosenau equation with hydrodynamical damped term," Journal of Mathematical Analysis and Applications, vol. 401, no. 2, pp. 763-773, 2013.

[15] H. W. Wang and S. B. Wang, "Decay and scattering of small solutions for Rosenau equations," Applied Mathematics and Computation, vol. 218, no. 1, pp. 115-123, 2011.

[16] L. C. Evance, Partial Differential Equations, American Mathematical Society, Providence, RI, USA, 1988.

[17] H. A. Levine, "Instability and nonexistence of global solutions to nonlinear wave equations of the form $P u_{t t}=-A u+\mathscr{F}(u)$," Transactions of the American Mathematical Society, vol. 192, pp. 1-21, 1974.

[18] J. L. Lions, Quelques Méthodes de Résolution des Problèmes aux Limites non Linéaires, Dunod, Paris, France, 1969.

[19] J. C. Robinson, Infinite-Dimensional Dynamical Systems, An Introduction to Dissipative Parabolic PDEs and the Theory of Global Attractors, Cambridge Texts in Applied Mathematics, Cambridge University Press, Cambridge, UK, 2001. 


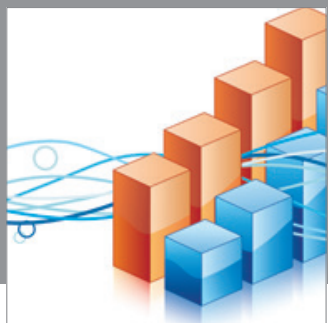

Advances in

Operations Research

mansans

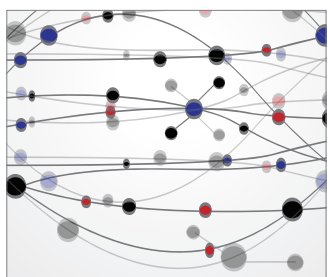

The Scientific World Journal
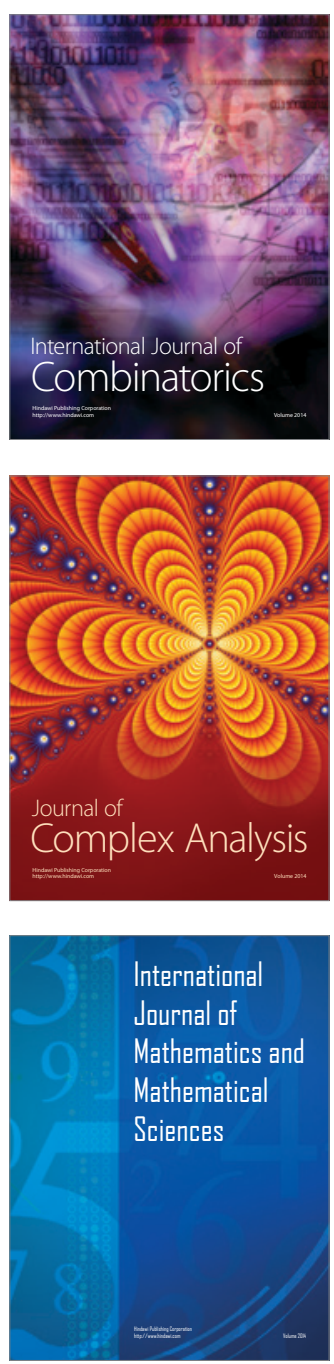
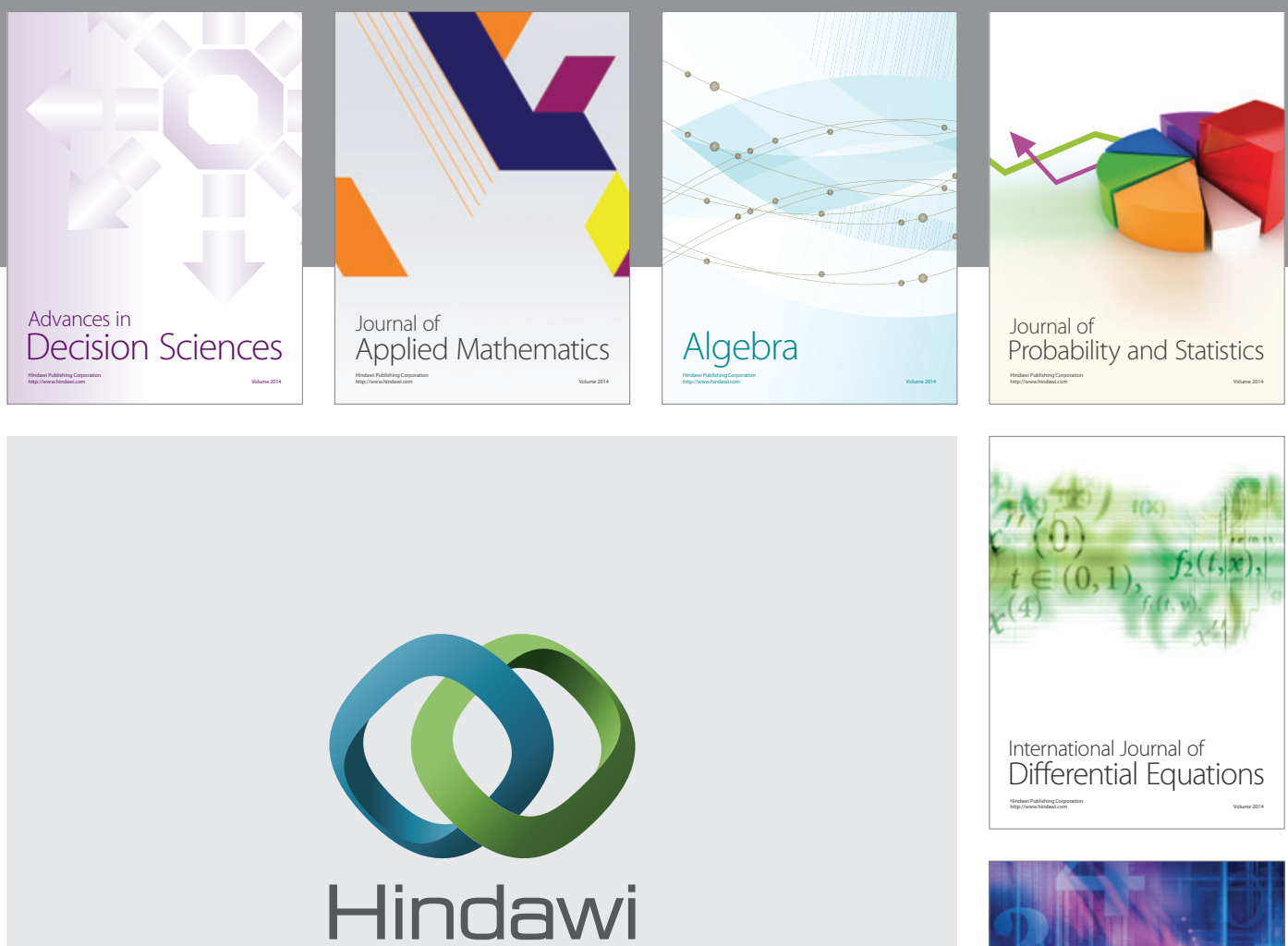

Submit your manuscripts at http://www.hindawi.com
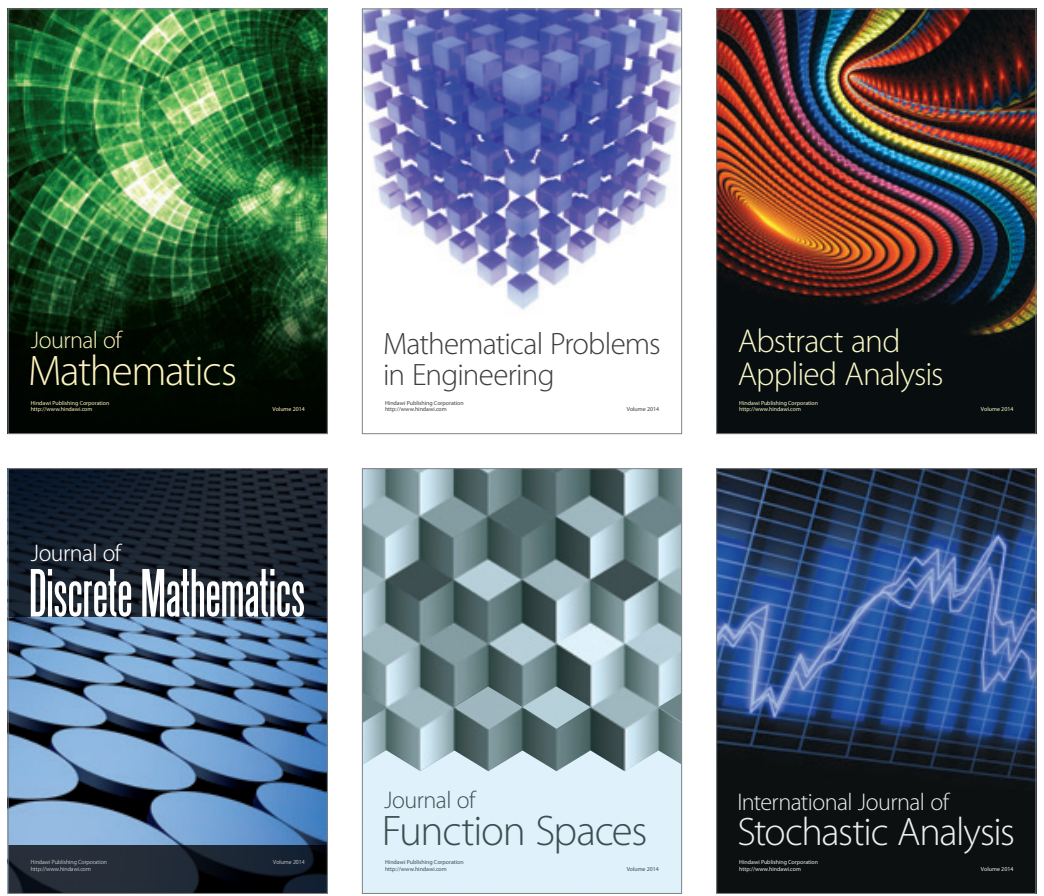

Journal of

Function Spaces

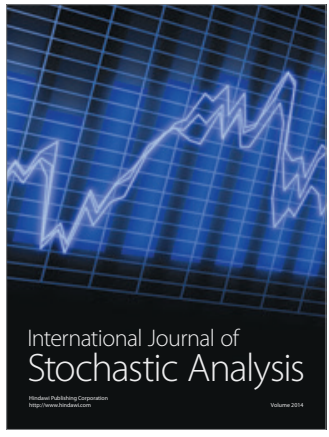

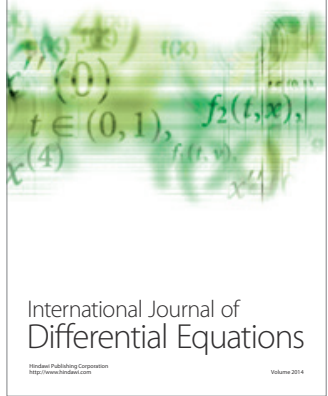
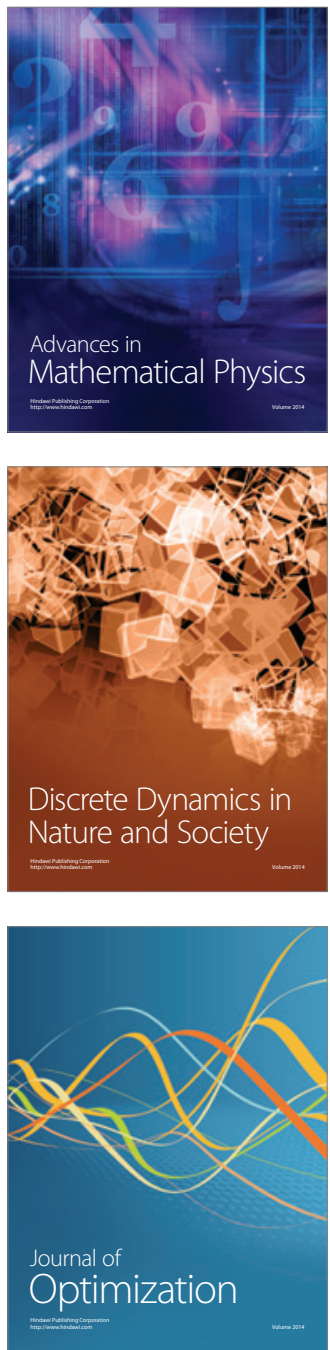